\title{
KÄHLERIAN SUBMERSIONS WITH VANISHING BOCHNER CURVATURE TENSOR
}

\author{
KAZUHIKO TAKANO
}

\begin{abstract}
In this paper, we discuss the Kählerian submersion with vanishing Bochner curvature tensor and prove that the Bochner curvature tensor of the base space vanishes. Also, we seek a sufficient condition with respect to the length of the Ricci tensor of each fiber that the Bochner curvature tensor of each fiber vanishes.
\end{abstract}

\section{Introduction}

Let $(M, g)$ and $(\widehat{M}, \widehat{g})$ be two Riemannian manifolds. A Riemannian submersion $\pi: M \rightarrow \widehat{M}$ is a mapping of $M$ onto $\widehat{M}$ such that $\pi$ has maximal rank and $\pi_{*}$ preserves the length of horizontal vectors. The various fundamental equations for Riemannian submersions were found by B. O'Neill [5].

Let $(M, g, \phi)$ be a real $2 m$-dimensional Kählerian manifold with the almost complex structure $\phi$ and Kählerian metric $g$, and $(\widehat{M}, \widehat{g}, \widehat{\phi})$ a real $2 n$-dimensional almost complex manifold with the almost complex structure $\hat{\phi}$ and metric $\widehat{g}$. A Riemannian submersion $\pi: M \rightarrow \widehat{M}$ is said to be a Kählerian submersion, if it commutes with the almost complex structures. B. Watson [9] has studied the Kählerian submersion and proved the following theorem:

Theorem A. Let $\pi: M \rightarrow \widehat{M}$ be a Kählerian submersion. Then the base space and each fiber are Kählerian manifolds, and the horizontal distribution is integrable. Moreover, if $M$ is of constant holomorphic sectional curvature $c$, then $\widehat{M}$ is a space of constant holomorphic sectional curvature $c(\leq 0)$.

On the other hand, Riemannian submersions with almost contact structure of contact structure is studied in [2] and [8]. Also, B. H. Kim [3] and the author [7] have investigated Riemannian subersions with Sasakian structure such that contact Bochner curvature tensor of the total space vanishes identically.

In and $\S 3$, we prepare fundamental equations of the Riemannian and Kählerian submersion, respectively. We consider the Kählerian submersion with vanishing Bochner curvature tensor in

Received November 25, 1998.

1991 Mathematics Subject Classification. Primary 53C55, Secondary 53C42.

Key words and phrases. Riemannian submersion, Kählerian submersion, Bochner curvature tensor. 
The author would like to express his hearty thanks to Professors S. Yamaguchi and N. Abe for his helpful advice.

\section{Riemannian Submersions}

Let $\pi: M \rightarrow \widehat{M}$ be a Riemannian submersion. We put $\operatorname{dim} M=m$ and $\operatorname{dim} \widehat{M}=n$. For each point $x \in \widehat{M}$, submanifolds $\pi^{-1}(x)$ with the induced metric $\bar{g}$ are called fibers and denoted by $\bar{M}_{x}$. We notice that the dimension of each fiber is always $m-n(=s)$. A vector field on $M$ is vertical if it is always tangent to fibers, horizontal if always orthogonal to fibers. We denote the vertical and horizontal subspace in the tangent space $T_{p}(M)$ of the total space $M$ by $\mathcal{V}_{p}(M)$ and $\mathcal{H}_{p}(M)$ for each point $p \in M$, and the vertical and horizontal distributions in the tangent bundle $T(M)$ of $M$ by $\mathcal{V}(M)$ and $\mathcal{H}(M)$, respectively. Then $T(M)$ is the direct sum of $\mathcal{V}(M)$ and $\mathcal{H}(M)$. The projection mappings are denoted $\mathcal{V}: T(M) \rightarrow \mathcal{V}(M)$ and $\mathcal{H}: T(M) \rightarrow \mathcal{H}(M)$ respectively. Also, we denote by $\nabla$ the Levi-Civita connection of $g$ and $\bar{\nabla}$ the collection of all Levi-Civita connections of $\bar{g}$. Notice that $\bar{\nabla}_{U} V$ is a well-defined vertical vector field on $M$ for vertical vector fields $U$ and $V$ on $M$, more precisely $\bar{\nabla}_{U} V=\mathcal{V} \nabla_{U} V$. The letters $U, V, W$, $W^{\prime}$ will always denote vertical vector fields, and $X, Y, Z, Z^{\prime}$ horizontal vector fields.

We define the (1,2)-tensor $T$ on $M$ for vector fields $E$ and $F$ by

$$
T_{E} F=\mathcal{H} \nabla_{\mathcal{V} E} \mathcal{V} F+\mathcal{V} \nabla_{\mathcal{V} E} \mathcal{H} F
$$

Then $T$ satisfies the following properties [5]:

(1) At each point, $T_{E}$ is a skew-symmetric linear operator on any $T_{p}(M)$, and it reverses the horizontal and vertical subspaces.

(2) $T$ is vertical; that is, $T_{E}=T_{\mathcal{V} E}$.

(3) For vertical vector fields, $T$ has the symmetry property $T_{U} V=T_{V} U$.

$T$ is related to the second fundamental form of fibers, it is identically zero if and only if each fiber is totally geodesic. We call the Riemannian submersion with totally geodesic fiber if $T$ vanishes identically.

Next, we define the integrability $(1,2)$-tensor $A$ associated with the submersion as follows:

$$
A_{E} F=\mathcal{H} \nabla_{\mathcal{H} E} \nabla \mathcal{V} F+\mathcal{V} \nabla_{\mathcal{H} E} \mathcal{H} F
$$

for vector fields $E$ and $F$. Then it has the following properties [5]:

$\left(1^{\prime}\right)$ At each point, $A_{E}$ is a skew-symmetric linear operator on $T_{p}(M)$, and it reverses the horizontal and vertical subspaces.

$\left(2^{\prime}\right) A$ is horizontal; that is, $A_{E}=A_{\mathcal{H} E}$.

$\left(3^{\prime}\right)$ For horizontal vector fields, $A$ has the alternation property $A_{X} Y=-A_{Y} X=$ $\frac{1}{2} \mathcal{V}[X, Y]$. 
Here, since $A$ is related to the integrability of $\mathcal{H}(M)$, it is identically zero if and only if $\mathcal{H}(M)$ is integrable. Moreover, if $A$ and $T$ vanish identically, then the total space is a locally product space of the base space and fiber.

We call a vector field $X$ on $M$ projectable if there exists a vector field $X_{*}$ on $\widehat{M}$ such that $\pi_{*}\left(X_{p}\right)=X_{* \pi(p)}$ for each $p \in M$, and say that $X$ and $X_{*}$ are $\pi$-related. Also, a vector field $X$ on $M$ is called basic if it is projectable and horizontal. Then we have [5]

Lemma B. If $X$ and $Y$ are basic vector field on $M$ which are $\pi$-related to $X_{*}$ and $Y_{*}$ on $\widehat{M}$, then

(1) $\widehat{g}\left(X_{*}, Y_{*}\right)=g(X, Y) \circ \pi$, where $g$ is the metric on $M$ and $\widehat{g}$ the metric on $\widehat{M}$,

(2) $\mathcal{H}[X, Y]$ is basic and is $\pi$-related to $\left[X_{*}, Y_{*}\right]$,

(3) $\mathcal{H} \nabla_{X} Y$ is basic and is $\pi$-related to $\widehat{\nabla}_{X_{*}} Y_{*}$, where $\widehat{\nabla}$ is the Riemannian connection of $\widehat{M}$.

Lemma C. Let $X$ and $Y$ be horizontal vector fields, $U$ and $V$ vertical vector fields. Then

$$
\begin{aligned}
\nabla_{U} V & =T_{U} V+\bar{\nabla}_{U} V, \\
\nabla_{U} X & =\mathcal{H} \nabla_{U} X+T_{U} X, \\
\nabla_{X} U & =A_{X} U+\mathcal{V} \nabla_{X} U, \\
\nabla_{X} Y & =\mathcal{H} \nabla_{X} Y+A_{X} Y .
\end{aligned}
$$

Furthermore, if $X$ is basic, $\mathcal{H} \nabla_{U} X=A_{X} U$.

Next, we denote by $R$ the curvature tensor of $g$, by $\bar{R}$ the collection of all curvature tensors of the Riemannian metric $\bar{g}$ on the fiber and by $\widehat{R}(X, Y) Z$ the horizontal vector field such that $\pi_{*}(\widehat{R}(X, Y) Z)=\widehat{R}\left(\pi_{*} X, \pi_{*} Y\right) \pi_{*} Z$ at each $p \in M$, where $\widehat{R}$ is the curvature tensor of $\widehat{g}$ on $\widehat{M}$. Then we have [5]

Lemma D. Let $U, V, W, W^{\prime}$ be vertical vector fields, and $X, Y, Z, Z^{\prime}$ horizontal vector fields. Then

$$
\begin{aligned}
g\left(R(U, V) W, W^{\prime}\right)= & g\left(\bar{R}(U, V) W, W^{\prime}\right)+g\left(T_{U} W, T_{V} W^{\prime}\right)-g\left(T_{V} W, T_{U} W^{\prime}\right), \\
g(R(U, V) W, X)= & g\left(\left(\nabla_{U} T\right)_{V} W, X\right)-g\left(\left(\nabla_{V} T\right)_{U} W, X\right), \\
g(R(X, U) Y, V)= & g\left(\left(\nabla_{X} T\right)_{U} Y, V\right)-g\left(\left(\nabla_{U} A\right)_{X} Y, V\right)+g\left(T_{U} X, T_{V} Y\right) \\
& -g\left(A_{X} U, A_{Y} V\right), \\
g(R(U, V) X, Y)= & g\left(\left(\nabla_{U} A\right)_{X} V, Y\right)-g\left(\left(\nabla_{V} A\right)_{X} U, Y\right)-g\left(A_{X} U, A_{Y} V\right) \\
& +g\left(A_{X} V, A_{Y} U\right)+g\left(T_{U} X, T_{V} Y\right)-g\left(T_{V} X, T_{U} Y\right), \\
g(R(X, Y) Z, U)= & -g\left(\left(\nabla_{Z} A\right)_{X} Y, U\right)-g\left(A_{X} Y, T_{U} Z\right)+g\left(A_{Y} Z, T_{U} X\right) \\
& +g\left(A_{Z} X, T_{U} Y\right), \\
g\left(R(X, Y) Z, Z^{\prime}\right)= & g\left(\widehat{R}(X, Y) Z, Z^{\prime}\right)+2 g\left(A_{X} Y, A_{Z} Z^{\prime}\right)-g\left(A_{Y} Z, A_{X} Z^{\prime}\right) \\
& +g\left(A_{X} Z, A_{Y} Z^{\prime}\right) .
\end{aligned}
$$


Also, in [5] we get

$$
g\left(\left(\nabla_{U} A\right)_{X} Y, V\right)+g\left(\left(\nabla_{V} A\right)_{X} Y, U\right)=g\left(\left(\nabla_{Y} T\right)_{U} V, X\right)-g\left(\left(\nabla_{X} T\right)_{U} V, Y\right) .
$$

For each $p \in M$, we denote by $\left\{X_{1}, \ldots, X_{n}\right\}$ and $\left\{U_{1}, \ldots, U_{s}\right\}$ local orthonormal basis of $\mathcal{H}(M)$ and $\mathcal{V}(M)$, respectively. Then we define [1]

$$
\begin{aligned}
g\left(A_{X}, A_{Y}\right) & =\sum_{i=1}^{n} g\left(A_{X} X_{i}, A_{Y} X_{i}\right)=\sum_{\alpha=1}^{s} g\left(A_{X} U_{\alpha}, A_{Y} U_{\alpha}\right) \\
g\left(A_{X}, T_{U}\right) & =\sum_{i=1}^{n} g\left(A_{X} X_{i}, T_{U} X_{i}\right)=\sum_{\alpha=1}^{s} g\left(A_{X} U_{\alpha}, T_{U} U_{\alpha}\right), \\
g\left(T_{U}, T_{V}\right) & =\sum_{i=1}^{n} g\left(T_{U} X_{i}, T_{V} X_{i}\right)=\sum_{\alpha=1}^{s} g\left(T_{U} U_{\alpha}, T_{V} U_{\alpha}\right), \\
g(A U, A V) & =\sum_{i=1}^{n} g\left(A_{X_{i}} U, A_{X_{i}} V\right) \\
g(T X, T Y) & =\sum_{\alpha=1}^{s} g\left(T_{U_{\alpha}} X, T_{U_{\alpha}} Y\right)
\end{aligned}
$$

and we put

$$
\begin{aligned}
& g\left(\left(\nabla_{U} T\right)_{V}, T_{W}\right)=\sum_{\alpha=1}^{s} g\left(\left(\nabla_{U} T\right)_{V} U_{\alpha}, T_{W} U_{\alpha}\right) \\
& g\left(\left(\nabla_{X} T\right) Y, T Z\right)=\sum_{\alpha=1}^{s} g\left(\left(\nabla_{X} T\right)_{U_{\alpha}} Y, T_{U_{\alpha}} Z\right) .
\end{aligned}
$$

Also, for tensors $A$ and $T$ we set

$$
\widehat{\delta} A=-\sum_{i=1}^{n}\left(\nabla_{X_{i}} A\right)_{X_{i}}, \quad \bar{\delta} T=-\sum_{\alpha=1}^{s}\left(\nabla_{U_{\alpha}} T\right)_{U_{\alpha}} .
$$

Moreover, we define the symmetric tensor $\widetilde{\delta} T$ by

$$
(\widetilde{\delta} T)(U, V)=\sum_{i=1}^{n} g\left(\left(\nabla_{X_{i}}, T\right)_{U} V, X_{i}\right)
$$

for vertical vector fields $U$ and $V$. Also, the mean curvature vector along each fiber gives the horizontal vector field

$$
N=\sum_{\alpha=1}^{s} T_{U_{\alpha}} U_{\alpha}
$$

If $N$ is identically zero, then each fiber is called a minimal submanifold of $M$. 
Let Ric, $\widehat{\text { Ric }}$ and $\overline{\text { Ric }}$ be the Ricci tensors of the Riemannian metrics $g, \widehat{g}$ and $\bar{g}$, respectively. Then we have [1]

$$
\begin{aligned}
\operatorname{Ric}(U, V) & =\overline{\operatorname{Ric}}(U, V)-g\left(N, T_{U} V\right)+g(A U, A V)+(\widetilde{\delta} T)(U, V) \\
\operatorname{Ric}(X, U) & =g((\bar{\delta} T) U, X)-g((\widehat{\delta} A) X, U)-2 g\left(A_{X}, T_{U}\right)+g\left(\nabla_{U} N, X\right) \\
\operatorname{Ric}(X, Y) & =\widehat{\operatorname{Ric}}(X, Y)-2 g\left(A_{X}, A_{Y}\right)-g(T X, T Y)+\frac{1}{2}\left\{g\left(\nabla_{X} N, Y\right)+g\left(\nabla_{Y} N, X\right)\right\}
\end{aligned}
$$

where $\widehat{\operatorname{Ric}}$ is the horizontal symmetric 2 -form on $M$ such that $\widehat{\operatorname{Ric}}(X, Y)=\widehat{\operatorname{Ric}}\left(\pi_{*} X, \pi_{*} Y\right)$. Moreover, if $r, \widehat{r}$ and $\bar{r}$ are the scalar curvatures of the Riemannian metrics $g, \widehat{g}$ and $\bar{g}$, respectively, then

$$
r=\widehat{r}+\bar{r}-2 \widehat{\delta} N-|N|^{2}-|A|^{2}-|T|^{2},
$$

where we denote $\widehat{r} \circ \pi$ by $\widehat{r}$ simply and we put $|N|^{2}=g(N, N)$,

$$
\begin{aligned}
& |A|^{2}=\sum_{i=1}^{n} g\left(A_{X_{i}}, A_{X_{i}}\right)=\sum_{\alpha=1}^{s} g\left(A U_{\alpha}, A U_{\alpha}\right), \\
& |T|^{2}=\sum_{i=1}^{n} g\left(T X_{i}, T X_{i}\right)=\sum_{\alpha=1}^{s} g\left(T_{U_{\alpha}}, T_{U_{\alpha}}\right) .
\end{aligned}
$$

\section{Kählerian Manifolds and Kählerian Submersions}

Let $(M, g, \phi)$ be a real $2 m$-dimensional Kählerian manifold with the almost complex structure $\phi$ and Kählerian metric $g$, that is, $\phi^{2}=-I, g$ and $\phi$ are compatible and $\nabla \phi=0$. For a Kählerian manifold, the Ricci tensor satisfies the following property:

$$
\operatorname{Ric}\left(\phi E_{1}, \phi E_{2}\right)=\operatorname{Ric}\left(E_{1}, E_{2}\right)
$$

for vector fields $E_{1}$ and $E_{2}$ on $M$. A Kählerian manifold $M$ is said to be a space of constant holomorphic sectional curvature $c$ if the curvature tensor satisfies

$$
\begin{aligned}
g\left(R\left(E_{1}, E_{2}\right) E_{3}, E_{4}\right)=\frac{c}{4}\{ & g\left(E_{2}, E_{3}\right) g\left(E_{1}, E_{4}\right)-g\left(E_{1}, E_{3}\right) g\left(E_{2}, E_{4}\right) \\
& +g\left(\phi E_{2}, E_{3}\right) g\left(\phi E_{1}, E_{4}\right)-g\left(\phi E_{1}, E_{3}\right) g\left(\phi E_{2}, E_{4}\right) \\
& \left.+2 g\left(E_{1}, \phi E_{2}\right) g\left(\phi E_{3}, E_{4}\right)\right\}
\end{aligned}
$$

for vector fields $E_{i}(i=1,2,3,4)$ on $M$. We easily find $c=\frac{r}{m(m+1)}$.

The Bochner curvature tensor $B$ of $M$ is defined by [6]

$$
\begin{aligned}
& g\left(B\left(E_{1}, E_{2}\right) E_{3}, E_{4}\right) \\
= & g\left(R\left(E_{1}, E_{2}\right) E_{3}, E_{4}\right) \\
& +\frac{1}{2 m+4}\left\{g\left(E_{1}, E_{3}\right) \operatorname{Ric}\left(E_{2}, E_{4}\right)-g\left(E_{2}, E_{3}\right) \operatorname{Ric}\left(E_{1}, E_{4}\right)+\operatorname{Ric}\left(E_{1}, E_{3}\right) g\left(E_{2}, E_{4}\right)\right.
\end{aligned}
$$




$$
\begin{aligned}
& -\operatorname{Ric}\left(E_{2}, E_{3}\right) g\left(E_{1}, E_{4}\right)+g\left(\phi E_{1}, E_{3}\right) S\left(E_{2}, E_{4}\right)-g\left(\phi E_{2}, E_{3}\right) S\left(E_{1}, E_{4}\right) \\
& +S\left(E_{1}, E_{3}\right) g\left(\phi E_{2}, E_{4}\right)-S\left(E_{2}, E_{3}\right) g\left(\phi E_{1}, E_{4}\right)+2 g\left(\phi E_{1}, E_{2}\right) S\left(E_{3}, E_{4}\right) \\
& \left.+2 S\left(E_{1}, E_{2}\right) g\left(\phi E_{3}, E_{4}\right)\right\} \\
& -\frac{r}{(2 m+4)(2 m+2)}\left\{g\left(E_{1}, E_{3}\right) g\left(E_{2}, E_{4}\right)-g\left(E_{2}, E_{3}\right) g\left(E_{1}, E_{4}\right)\right. \\
& +g\left(\phi E_{1}, E_{3}\right) g\left(\phi E_{2}, E_{4}\right)-g\left(\phi E_{2}, E_{3}\right) g\left(\phi E_{1}, E_{4}\right) \\
& \left.+2 g\left(\phi E_{1}, E_{2}\right) g\left(\phi E_{3}, E_{4}\right)\right\}
\end{aligned}
$$

for vector fields $E_{i}(i=1,2,3,4)$ on $M$, where $S\left(E_{1}, E_{2}\right)=\operatorname{Ric}\left(\phi E_{1}, E_{2}\right)$.

Next, we put $(\widehat{M}, \widehat{g}, \widehat{\phi})$ is a real $2 n$-dimensional almost complex manifold with the almost complex structure $\widehat{\phi}$ and metric $\widehat{g}$. A Riemannian submersion $\pi: M \rightarrow \widehat{M}$ is said to be a Kählerian submersion if $\pi_{*} \phi=\widehat{\phi} \pi_{*}$. Then B. Watson [9] proved that the vertical and horizontal distributions determined by $\pi$ are $\phi$-invariant, that is, $\phi \mathcal{V}(M)=\mathcal{V}(M)$ and $\phi \mathcal{H}(M)=\mathcal{H}(M)$. If $X$ is basic on $M$ which is $\pi$-related to $X_{*}$ on $\widehat{M}$, then $\phi X$ is basic and $\pi$-related to $\widehat{\phi} X_{*}$. We find from Theorem A that a given property on $M$ is induced onto $\widehat{M}$ and onto $\bar{M}_{x}$ for each point $x \in \widehat{M}$ by $\pi$. Thus, we denote the induced almost complex structure of each fiber by $\bar{\phi}$. We put $\operatorname{dim} \bar{M}_{x}=2 s(m=n+s)$. Also, a Kählerian submersion is said to be with vanishing Bochner curvature tensor if the Bochner curvature tensor of $M$ vanishes identically.

We will seek the fundamental equations of the Kählerian submersion. Let $\pi: M \rightarrow \widehat{M}$ be a Kählerian submersion. Then the integrability tensor $A$ vanishes from Theorem A. Also, it is easy to see from Lemma $\mathrm{C}$ that $\nabla \phi=0$ is equivalent to the following equations:

$$
\begin{aligned}
\left(\mathcal{H} \nabla_{X} \phi\right) Y & =0, \\
\left(\mathcal{V} \nabla_{X} \bar{\phi}\right) U & =0, \\
\left(\mathcal{H} \nabla_{U} \phi\right) X & =0, \\
T_{U}(\phi X) & =\bar{\phi}\left(T_{U} X\right), \\
T_{U}(\bar{\phi} V) & =\phi\left(T_{U} V\right), \\
\left(\bar{\nabla}_{U} \bar{\phi}\right) V & =0
\end{aligned}
$$

for horizontal vector fields $X$ and $Y$, vertical vector fields $U$ and $V$. Since each fiber is invariant submanifold of $M$, each fiber is minimal, that is, $N=0$. Therefore, equations (2.5)-(2.10) and (2.11) are rewritten as follows:

$$
\begin{aligned}
g\left(R(U, V) W, W^{\prime}\right) & =g\left(\bar{R}(U, V) W, W^{\prime}\right)+g\left(T_{U} W, T_{V} W^{\prime}\right)-g\left(T_{V} W, T_{U} W^{\prime}\right), \\
g(R(U, V) W, X) & =g\left(\left(\nabla_{U} T\right)_{V} W, X\right)-g\left(\left(\nabla_{V} T\right)_{U} W, X\right), \\
g(R(X, U) Y, V) & =g\left(\left(\nabla_{X} T\right)_{U} Y, V\right)+g\left(T_{U} X, T_{V} Y\right), \\
g(R(U, V) X, Y) & =g\left(T_{U} X, T_{V} Y\right)-g\left(T_{V} X, T_{U} Y\right), \\
g(R(X, Y) Z, U) & =0 \\
g\left(R(X, Y) Z, Z^{\prime}\right) & =g\left(\widehat{R}(X, Y) Z, Z^{\prime}\right)
\end{aligned}
$$


and

$$
g\left(\left(\nabla_{Y} T\right)_{U} V, X\right)-g\left(\left(\nabla_{X} T\right)_{U} V, Y\right)=0 .
$$

Let $\left\{X_{1}, \ldots, X_{2 n}, U_{1}, \ldots, U_{2 s}\right\}$ be a local orthonormal frame such that $\left\{X_{1}, \ldots, X_{2 n}\right\}$ and $\left\{U_{1}, \ldots, U_{2 s}\right\}$ are local orthonormal bases of $\mathcal{H}(M)$ and $\mathcal{V}(M)$, respectively, where $X_{n+i}=\phi X_{i}(i=1,2, \ldots, n)$ and $U_{s+\alpha}=\bar{\phi} U_{\alpha}(\alpha=1,2, \ldots, s)$. By virtue of $A=0$ and $N=0$, we get from (2.12)-(2.14) and (2.15)

$$
\begin{aligned}
\operatorname{Ric}(U, V) & =\overline{\operatorname{Ric}}(U, V)+(\widetilde{\delta} T)(U, V), \\
\operatorname{Ric}(X, U) & =g((\bar{\delta} T) U, X), \\
\operatorname{Ric}(X, Y) & =\widehat{\operatorname{Ric}}(X, Y)-g(T X, T Y)
\end{aligned}
$$

and

$$
r=\widehat{r}+\bar{r}-|T|^{2} .
$$

Also, we define skew-symmetric tensors $S, \widehat{S}$ and $\bar{S}$ by $S(E, F)=\operatorname{Ric}(\phi E, F)$, $\widehat{S}(X, Y)=\widehat{\operatorname{Ric}}(\phi X, Y)$ and $\bar{S}(U, V)=\overline{\operatorname{Ric}}(\bar{\phi} U, V)$, respectively. Then we obtain from (3.17)-(3.19) that

$$
\begin{aligned}
S(U, V) & =\bar{S}(U, V)+(\widetilde{\delta} T)(\bar{\phi} U, V), \\
S(X, U) & =g((\bar{\delta} T) U, \phi X), \\
S(X, Y) & =\widehat{S}(X, Y)-g(T \phi X, T Y) .
\end{aligned}
$$

From $(3.21)$, we find $(\widetilde{\delta} T)(U, V)-(\widetilde{\delta} T)(\bar{\phi} U, \bar{\phi} V)=0$. On the other hand, by a straightforward computation we have $(\widetilde{\delta} T)(\bar{\phi} U, \bar{\phi} V)=-(\widetilde{\delta} T)(U, V)$, where we have used (3.5) and (3.8). Thus we get

$$
(\widetilde{\delta} T)(U, V)=0 .
$$

From (3.17) and (3.24), we have

Lemma 3.1. Let $\pi: M \rightarrow \widehat{M}$ be a Kählerian submersion. If the total space is of constant holomorphic sectional curvature, then each fiber is an Einstein manifold.

\section{Kählerian Submersions with Vanishing Bochner Curvature Tensor}

In this section, we shall consider the Kählerian submersion $\pi: M \rightarrow \widehat{M}$ with vanishing Bochner curvature tensor. Then we see from (3.3), (3.10)-(3.15), (3.17)-(3.19), (3.21)(3.23) that $g\left(B\left(E_{1}, E_{2}\right) E_{3}, E_{4}\right)=0$ is equivalent to the following equations for horizontal vector fields $X, Y, Z, Z^{\prime}$ and vertical vector fields $U, V, W, W^{\prime}$ :

$$
\begin{aligned}
g\left(\bar{R}(U, V) W, W^{\prime}\right)+g\left(T_{U} W, T_{V} W^{\prime}\right)-g\left(T_{V} W, T_{U} W^{\prime}\right) \\
\frac{1}{2 m+4}\left\{g(U, W) \overline{\operatorname{Ric}}\left(V, W^{\prime}\right)-g(V, W) \overline{\operatorname{Ric}}\left(U, W^{\prime}\right)+\overline{\operatorname{Ric}}(U, W) g\left(V, W^{\prime}\right)\right. \\
-\overline{\operatorname{Ric}}(V, W) g\left(U, W^{\prime}\right)+g(\bar{\phi} U, W) \bar{S}\left(V, W^{\prime}\right)-g(\bar{\phi} V, W) \bar{S}\left(U, W^{\prime}\right)
\end{aligned}
$$




$$
\begin{aligned}
& +\bar{S}(U, W) g\left(\bar{\phi} V, W^{\prime}\right)-\bar{S}(V, W) g\left(\bar{\phi} U, W^{\prime}\right)+2 g(\bar{\phi} U, V) \bar{S}\left(W, W^{\prime}\right) \\
& \left.+2 \bar{S}(U, V) g\left(\bar{\phi} W, W^{\prime}\right)\right\} \\
& -\frac{r}{(2 m+4)(2 m+2)}\left\{g(U, W) g\left(V, W^{\prime}\right)-g(V, W) g\left(U, W^{\prime}\right)+g(\bar{\phi} U, W) g\left(\bar{\phi} V, W^{\prime}\right)\right. \\
& \left.-g(\bar{\phi} V, W) g\left(\bar{\phi} U, W^{\prime}\right)+2 g(\bar{\phi} U, V) g\left(\bar{\phi} W, W^{\prime}\right)\right\}=0, \\
& g\left(\left(\nabla_{U} T\right)_{V} W, X\right)-g\left(\left(\nabla_{V} T\right)_{U} W, X\right) \\
& +\frac{1}{2 m+4}\{g(U, W) g((\bar{\delta} T) V, X)-g(V, W) g((\bar{\delta} T) U, X)+g(\bar{\phi} U, W) g((\bar{\delta} T) V, \phi X) \\
& -g(\bar{\phi} V, W) g((\bar{\delta} T) U, \phi X)+2 g(\bar{\phi} U, V) g((\bar{\delta} T) W, \phi X)\}=0 \\
& g\left(\left(\nabla_{X} T\right)_{U} Y, V\right)+g\left(T_{U} X, T_{V} Y\right) \\
& +\frac{1}{2 m+4}[g(X, Y) \overline{\operatorname{Ric}}(U, V)+\{\widehat{\operatorname{Ric}}(X, Y)-g(T X, T Y)\} g(U, V) \\
& +g(\phi X, Y) \bar{S}(U, V)+\{\widehat{S}(X, Y)-g(T \phi X, T Y)\} g(\bar{\phi} U, V)] \\
& -\frac{r}{(2 m+4)(2 m+2)}\{g(X, Y) g(U, V)+g(\phi X, Y) g(\bar{\phi} U, V)\}=0, \\
& g\left(T_{U} X, T_{V} Y\right)-g\left(T_{V} X, T_{U} Y\right)-\frac{2 r}{(2 m+4)(2 m+2)} g(\phi X, Y) g(\bar{\phi} U, V) \\
& +\frac{2}{2 m+4}[g(\phi X, Y) \bar{S}(U, V)+\{\widehat{S}(X, Y)-g(T \phi X, T Y)\} g(\bar{\phi} U, V)]=0, \\
& g(X, Z) g((\bar{\delta} T) U, Y)-g(Y, Z) g((\bar{\delta} T) U, X) \\
& +g(\phi X, Z) g((\bar{\delta} T) U, \phi Y)-g(\phi Y, Z) g((\bar{\delta} T) U, \phi X)+2 g(\phi X, Y) g((\bar{\delta} T) U, \phi Z)=0,(4.5) \\
& g\left(\widehat{R}(X, Y) Z, Z^{\prime}\right) \\
& +\frac{1}{2 m+4}\left[g(X, Z)\left\{\widehat{\operatorname{Ric}}\left(Y, Z^{\prime}\right)-g\left(T Y, T Z^{\prime}\right)\right\}-g(Y, Z)\left\{\widehat{\operatorname{Ric}}\left(X, Z^{\prime}\right)-g\left(T X, T Z^{\prime}\right)\right\}\right. \\
& +\{\widehat{\operatorname{Ric}}(X, Z)-g(T X, T Z)\} g\left(Y, Z^{\prime}\right)-\{\widehat{\operatorname{Ric}}(Y, Z)-g(T Y, T Z)\} g\left(X, Z^{\prime}\right) \\
& +g(\phi X, Z)\left\{\widehat{S}\left(Y, Z^{\prime}\right)-g\left(T \phi Y, T Z^{\prime}\right)\right\}-g(\phi Y, Z)\left\{\widehat{S}\left(X, Z^{\prime}\right)-g\left(T \phi X, T Z^{\prime}\right)\right\} \\
& +\{\widehat{S}(X, Z)-g(T \phi X, T Z)\} g\left(\phi Y, Z^{\prime}\right)-\{\widehat{S}(Y, Z)-g(T \phi Y, T Z)\} g\left(\phi X, Z^{\prime}\right) \\
& \left.+2 g(\phi X, Y)\left\{\widehat{S}\left(Z, Z^{\prime}\right)-g\left(T \phi Z, T Z^{\prime}\right)\right\}+2\{\widehat{S}(X, Y)-g(T \phi X, T Y)\} g\left(\phi Z, Z^{\prime}\right)\right] \\
& -\frac{r}{(2 m+4)(2 m+2)}\left\{g(X, Z) g\left(Y, Z^{\prime}\right)-g(Y, Z) g\left(X, Z^{\prime}\right)+g(\phi X, Z) g\left(\phi Y, Z^{\prime}\right)\right. \\
& \left.-g(\phi Y, Z) g\left(\phi X, Z^{\prime}\right)+2 g(\phi X, Y) g\left(\phi Z, Z^{\prime}\right)\right\}=0 .
\end{aligned}
$$

From (4.5), we get

$$
g((\bar{\delta} T) U, X)=0 .
$$


Substituting this into (4.2), we find

$$
g\left(\left(\nabla_{U} T\right)_{V} W, X\right)-g\left(\left(\nabla_{V} T\right)_{U} W, X\right)=0 .
$$

Because of (3.20), we see from (4.6) that

$$
s \widehat{\operatorname{Ric}}(Y, Z)+(n+2) g(T Y, T Z)-\frac{s\left(\widehat{r}-|T|^{2}\right)-(n+1) \bar{r}}{2(n+s+1)} g(Y, Z)=0
$$

which yields

$$
\frac{\widehat{r}}{n(n+1)}+\frac{\bar{r}}{s(s+1)}+\frac{n+2 s+2}{n s(s+1)}|T|^{2}=0 .
$$

If we substitute (4.10) into (4.9), then we find

$$
g(T Y, T Z)=-\frac{s}{n+2} \widehat{\operatorname{Ric}}(Y, Z)+\frac{s \widehat{r}+(n+2)|T|^{2}}{2 n(n+2)} g(Y, Z) .
$$

Owing to (3.20) and (4.11), equation (4.6) can be rewritten as follows:

$$
\begin{aligned}
& g\left(\widehat{R}(X, Y) Z, Z^{\prime}\right) \\
& +\frac{1}{2 n+4}\left\{\begin{array}{l}
g(X, Z) \widehat{\operatorname{Ric}}\left(Y, Z^{\prime}\right)-g(Y, Z) \widehat{\operatorname{Ric}}\left(X, Z^{\prime}\right)+\widehat{\operatorname{Ric}}(X, Z) g\left(Y, Z^{\prime}\right) \\
\quad \widehat{\operatorname{Ric}}(Y, Z) g\left(X, Z^{\prime}\right)+g(\phi X, Z) \widehat{S}\left(Y, Z^{\prime}\right)-g(\phi Y, Z) \widehat{S}\left(X, Z^{\prime}\right) \\
\quad+\widehat{S}(X, Z) g\left(\phi Y, Z^{\prime}\right)-\widehat{S}(Y, Z) g\left(\phi X, Z^{\prime}\right)+2 g(\phi X, Y) \widehat{S}\left(Z, Z^{\prime}\right) \\
\left.\quad+2 \widehat{S}(X, Y) g\left(\phi Z, Z^{\prime}\right)\right\} \\
-\frac{\widehat{r} \quad}{(2 n+2)(2 n+4)}\left\{g(X, Z) g\left(Y, Z^{\prime}\right)-g(Y, Z) g\left(X, Z^{\prime}\right)+g(\phi X, Z) g\left(\phi Y, Z^{\prime}\right)\right. \\
\left.\quad-g(\phi Y, Z) g\left(\phi X, Z^{\prime}\right)+2 g(\phi X, Y) g\left(\phi Z, Z^{\prime}\right)\right\}=0 .
\end{array}\right.
\end{aligned}
$$

Hence, we have

Theorem 4.1. If $\pi: M \rightarrow \widehat{M}$ is a Kählerian submersion with vanishing Bochner curvature tensor, then the Bochner curvature tensor of $\widehat{M}$ vanishes.

From (4.10), we have

Lemma 4.1. Let $\pi: M \rightarrow \widehat{M}$ be a Kählerian submersion with vanishing Bochner curvature tensor. Then we get $\frac{\widehat{r}}{n(n+1)}+\frac{\bar{r}}{s(s+1)} \leq 0$, equality holds if and only if the Kählerian submersion has totally geodesic fiber.

Operating $\nabla_{X}$ to $(4.11)$, we find

$$
\begin{aligned}
& s\left(\nabla_{X} \widehat{\operatorname{Ric}}\right)(Y, Z)+(n+2)\left\{g\left(\left(\nabla_{X} T\right) Y, T Z\right)+g\left(T Y,\left(\nabla_{X} T\right) Z\right)\right\} \\
& -\frac{1}{2 n}\left\{s \nabla_{X} \widehat{r}+(n+2) \nabla_{X}|T|^{2}\right\} g(Y, Z)=0,
\end{aligned}
$$


which implies

$$
s \nabla_{Z} \widehat{r}+(n+2) \nabla_{Z}|T|^{2}=0
$$

if $n>1$. Thus we have

Lemma 4.2. Let $\pi: M \rightarrow \widehat{M}$ be a Kählerian submersion with vanishing Bochner curvature tensor. Then the scalar function $\widehat{s} \widehat{r}+(n+2)|T|^{2}$ is constant on $\widehat{M}$ if $n>1$.

Also, making use of $(3.20),(4.10)$ and $N=0$, we find from (4.1)

$$
n \overline{\operatorname{Ric}}(V, W)+(n+s+2) g\left(T_{V}, T_{W}\right)-\frac{1}{2 s}\left\{n \bar{r}+(n+s+2)|T|^{2}\right\} g(V, W)=0 .
$$

Operation $\nabla_{U}$ to the above equation, we obtain

$$
\begin{aligned}
& n\left(\nabla_{U} \overline{\mathrm{Ric}}\right)(V, W)+(n+s+2)\left\{g\left(\left(\nabla_{U} T\right)_{V}, T_{W}\right)+g\left(\left(\nabla_{U} T\right)_{W}, T_{V}\right)\right\} \\
& -\frac{1}{2 s}\left\{n \nabla_{U} \bar{r}+(n+s+2) \nabla_{U}|T|^{2}\right\} g(V, W)=0,
\end{aligned}
$$

which together with (4.7) and (4.8) implies that

$$
n \nabla_{V} \bar{r}+(n+s+2) \nabla_{V}|T|^{2}=0
$$

if $s>1$. Thus we have

Lemma 4.3. Let $\pi: M \rightarrow \widehat{M}$ be a Kählerian submersion with vanishing Bochner curvature tensor. Then the scalar function $n \bar{r}+(n+s+2)|T|^{2}$ is constant on each fiber if $s>1$.

From (4.14) we have

Lemma 4.4. Let $\pi: M \rightarrow \widehat{M}$ be a Kählerian submersion with vanishing Bochner curvature tensor. Each fiber is an Einstein manifold if and only if $g\left(T_{V}, T_{W}\right)=\frac{|T|^{2}}{2 s} g(V, W)$ if $s>1$.

Next, we consider the following Bochner curvature tensor $\bar{B}$ of each fiber

$$
\begin{aligned}
& g\left(\bar{B}(U, V) W, W^{\prime}\right) \\
& =g\left(\bar{R}(U, V) W, W^{\prime}\right) \\
& +\frac{1}{2 s+4}\left\{g(U, W) \overline{\operatorname{Ric}}\left(V, W^{\prime}\right)-g(V, W) \overline{\operatorname{Ric}}\left(U, W^{\prime}\right)+\overline{\operatorname{Ric}}(U, W) g\left(V, W^{\prime}\right)\right. \\
& -\overline{\operatorname{Ric}}(V, W) g\left(U, W^{\prime}\right)+g(\bar{\phi} U, W) \bar{S}\left(V, W^{\prime}\right)-g(\bar{\phi} V, W) \bar{S}\left(U, W^{\prime}\right) \\
& +\bar{S}(U, W) g\left(\bar{\phi} V, W^{\prime}\right)-\bar{S}(V, W) g\left(\bar{\phi} U, W^{\prime}\right)+2 g(\bar{\phi} U, V) \bar{S}\left(W, W^{\prime}\right) \\
& \left.+2 \bar{S}(U, V) g\left(\bar{\phi} W, W^{\prime}\right)\right\} \\
& -\frac{\bar{r}}{(2 s+2)(2 s+4)}\left\{g(U, W) g\left(V, W^{\prime}\right)-g(V, W) g\left(U, W^{\prime}\right)+g(\bar{\phi} U, W) g\left(\bar{\phi} V, W^{\prime}\right)\right. \\
& \left.-g(\bar{\phi} V, W) g\left(\bar{\phi} U, W^{\prime}\right)+2 g(\bar{\phi} U, V) g\left(\bar{\phi} W, W^{\prime}\right)\right\} .
\end{aligned}
$$


Substituting (4.1) into the above equation and owing to (3.20) and (4.10), we get

$$
\begin{aligned}
& g\left(\bar{B}(U, V) W, W^{\prime}\right) \\
& =-g\left(T_{U} W, T_{V} W^{\prime}\right)+g\left(T_{V} W, T_{U} W^{\prime}\right) \\
& +\frac{n}{2(s+2)(n+s+2)}\left\{g(U, W) \overline{\operatorname{Ric}}\left(V, W^{\prime}\right)-g(V, W) \overline{\operatorname{Ric}}\left(U, W^{\prime}\right)+\overline{\operatorname{Ric}}(U, W) g\left(V, W^{\prime}\right)\right. \\
& -\overline{\operatorname{Ric}}(V, W) g\left(U, W^{\prime}\right)+g(\bar{\phi} U, W) \bar{S}\left(V, W^{\prime}\right)-g(\bar{\phi} V, W) \bar{S}\left(U, W^{\prime}\right) \\
& +\bar{S}(U, W) g\left(\bar{\phi} V, W^{\prime}\right)-\bar{S}(V, W) g\left(\bar{\phi} U, W^{\prime}\right)+2 g(\bar{\phi} U, V) \bar{S}\left(W, W^{\prime}\right) \\
& \left.+2 \bar{S}(U, V) g\left(\bar{\phi} W, W^{\prime}\right)\right\} \\
& -\frac{1}{2 s}\left\{\frac{n \bar{r}}{(s+2)(n+s+2)}+\frac{|T|^{2}}{2(s+1)}\right\}\left\{g(U, W) g\left(V, W^{\prime}\right)-g(V, W) g\left(U, W^{\prime}\right)\right. \\
& \left.+g(\bar{\phi} U, W) g\left(\bar{\phi} V, W^{\prime}\right)-g(\bar{\phi} V, W) g\left(\bar{\phi} U, W^{\prime}\right)+2 g(\bar{\phi} U, V) g\left(\bar{\phi} W, W^{\prime}\right)\right\} \text {. }
\end{aligned}
$$

Denote by $|\bar{B}|^{2}$ the length of the Bochner curvature tensor of each fiber. Then making use of $(3.1),(3.7),(4.14)$ and $N=0$, we can get from the above equation

$$
\begin{aligned}
|\bar{B}|^{2}= & \sum_{\alpha, \beta=1}^{2 s}\left|\left[T_{U_{\alpha}}, T_{U_{\beta}}\right]\right|^{2}-\frac{8 n^{2}}{(s+2)(n+s+2)^{2}}|\overline{\mathrm{Ric}}|^{2} \\
& +\frac{4 n^{2}}{s(s+2)(n+s+2)^{2}} \bar{r}^{2}-\frac{2}{s(s+1)}|T|^{4} .
\end{aligned}
$$

Also, it is know that

Lemma E. ([10] p. 185) For a 2 s-dimensional Kählerian submanifold of a Kählerian manifold, the following inequality holds:

$$
\frac{1}{s}|T|^{4} \leq \sum_{\alpha, \beta=1}^{2 s}\left|\left[T_{U_{\alpha}}, T_{U_{\beta}}\right]\right|^{2} \leq|T|^{4} .
$$

From (4.16) and Lemma $E$, we have

Theorem 4.2. Let $\pi: M \rightarrow \widehat{M}$ be a Kählerian submersion with vanishing Bochner curvature tensor. If

$$
|\overline{\mathrm{Ric}}|^{2} \geq \frac{\bar{r}^{2}}{2 s}+\frac{(s-1)(s+2)}{8 s(s+1)}\left(\frac{n+s+2}{n}\right)^{2}|T|^{4},
$$

then the Bochner curvature tensor of each fiber vanishes identically.

Remark 4.1. Let $\pi: M \rightarrow \widehat{M}$ be a Kählerian submersion with vanishing Bochner curvature tensor. Then the length of the Ricci tensor satisfies the following inequality from (4.14)

$$
|\overline{\mathrm{Ric}}|^{2} \leq \frac{\bar{r}^{2}}{2 s}+\frac{2 s-1}{2 s}\left(\frac{n+s+2}{n}\right)^{2}|T|^{4}
$$


Remark 4.2. Let $\pi: M \rightarrow \widehat{M}$ be a Kählerian submersion with vanishing Bochner curvature tensor and totally geodesic fiber. Then $M$ is locally a product of two spaces of constant holomorphic sectional curvatures $c$ and $-c$, where $c=\frac{\widehat{r}}{n(n+1)}=\frac{\bar{r}}{s(s+1)}([4])$.

\section{References}

[1] A. Besse, Einstein Manifolds, Springer-Verlag, 1987.

[2] B. H. Kim, Fibred Riemannian spaces with contact structure, Hiroshima Math. J., 18 (1988), 493-508.

[3] —_, Fibred Sasakian spaces with vanishing contact Bochner curvature tensor, ibid., 19 (1989), 181-195.

[4] M. Matsumoto and S. Tanno, Kählerian spaces with parallel or vanishing Bochner curvature tensor, Tensor N. S., 27(1973), 291-294.

[5] B. O'Neill, The fundamental equations of a submersion, Michigan Math. J., 13 (1966), 459-469.

[6] S. Tachibana, On the Bochner curvature tensor, Natur. Sci. Rep. Ochanomizu Univ., 18(1967), 15-19.

[7] K. Takano, On fibred Sasakian spaces with vanishing contact Bochner curvature tensor, Colloq. Math., 65(1993), 181-200.

[8] Y. Tashiro and B. H. Kim, Almost complex and almost contact structures in fibred Riemannian spaces, Hiroshiam Math. J., 18 (1988), 161-188.

[9] B. Watson, Almost Hermitian submersion, J. Differential Geometry, 11(1976), 147-165.

[10] K. Yano and M. Kon, Structures on Manifolds, World Scientific, 1984.

Department of Mathematics, Faculty of Engineering, Shinshu University, 4-17-1 Wakasato, Nagano 380-8553, Japan.

E-mail: ktakano@gipwc.shinshu-u.ac.jp 